\title{
Robotic Insertion of Timber Joints using Visual Detection of Fiducial Markers
}

\author{
N. Rogeau ${ }^{\mathrm{a}}$, V. Tiberghien ${ }^{\mathrm{a}}$, P. Latteur ${ }^{\mathrm{b}}$ and Y. Weinand ${ }^{\mathrm{a}}$ \\ ${ }^{a}$ Laboratory for Timber Constructions, Ecole Polytechnique Fédérale de Lausanne, Switzerland \\ ${ }^{b}$ Louvain School of Engineering, Université Catholique de Louvain, Belgium \\ E-mail: nicolas.rogeau@epfl.ch
}

\begin{abstract}
-
Timber building industry is facing a major transformation with digitalization and automation being more broadly adopted. Prefabricated timber frame structures can be mass-produced on large robotic assembly lines, increasing the productivity and competitiveness of wood over standard inorganic materials such as steel or concrete. While standardization is likely to limit creativity, new digital tools, on the contrary, give the possibility to design and build complex and unique geometries. Recent research in bespoke digital prefabrication notably led to the development of Integrally Attached Timber Plate Structures (IATPS). This system consists in assembling wooden panels connected only with timber joints inspired by traditional Japanese carpentry. The elements are digitally prefabricated and inserted into one-another to form bespoke architectural structures. In order to propose a fully automated process for IATPS, from design to construction, this paper investigates a method for assembling the panels with a 6 -axis robotic arm. Preliminary studies have shown that significant discrepancies can occur between virtual models and physical prototypes due to joint tolerances, hygrometric variations, and self-weight deformations. To address this challenge and to adapt the robot position to the actual location of the elements, a visual feedback loop was developed using fiducial markers. Several tests were performed with structural wood panels to assess the accuracy of the method for different configurations and adapt the geometry of the joints in consequence. Finally, the insertion of a panel with two through-tenon joints was achieved by taking pictures of the target with a camera mounted on the end-effector of the robot.
\end{abstract}

\footnotetext{
Keywords -

Robotic assembly; Timber joints; Fiducial markers; Insertion
}

\section{Introduction}

The construction sector is recognized as one of the main players in the current ecological crisis, as the production of new construction materials is responsible for a significant share of $\mathrm{CO}_{2}$ emissions and plays a major role in the generation of landfill waste. In order to reach the Sustainable Development Goals set by the United Nations for 2030 [1], it is necessary to reconsider the whole construction process and take material life cycles into account upstream in the design phase.

Engineered wood products have been identified as an alternative to commonly used concrete and steel components, which could lead to more sustainable construction systems by lowering the embodied carbon energy of the structure [2]. In addition, connections between timber panels can be integrated into the element geometry taking inspiration from traditional timber joinery techniques and benefiting from recent advances in digital fabrication to generate the toolpath [3]. This construction system, also referred to as IntegrallyAttached Timber Plate Structures (IATPS), reduces the number of steel fasteners and improve the structural performance. Since additional connectors are not required, the amount of time allowed to the construction phase can also be reduced.

Previous research has both demonstrated the architectural and structural interest of IATPS, leading to the realization of large-scale projects such as the theater of Vidy [4], the BUGA wood pavilion [5], and the Annen head office [6]. Different workflows have been set up for each of those projects in order to integrate fabrication constraints in the design and automate the cutting of the different pieces using a $\mathrm{CNC}$ machine or a 6-axis robotic arm. For the BUGA wood pavilion, collaborative robots have also been used to glue the construction components. However, for each of the three projects, the assembly of the different modules remained a manual process. 
First investigations about the robotic assembly of IATPS have highlighted two main challenges for automating the insertion of timber joints [7]. First, friction forces are growing with the number of connections and can hinder the insertion. Second, the robotic insertion has to be performed with enough precision to avoid the introduction of gaps, which would decrease the rigidity of the connections. This paper focuses on the development of a method combining different strategies to automate the insertion of the panels.

\section{State of the art of robotic insertion}

Pick and place operations are usual tasks, which can be handled by industrial robotic arms. If the initial and final positions of the objects relative to the robot are known, the trajectory can be easily computed and the precision of the insertion will only depend on the accuracy of the robot from point to point. However, for large and heavy construction elements, significant discrepancies can occur between virtual models and reality. Timber panels are typically subject to slight dimensional changes over time and are very sensitive to hygrometric variations. Even for standardized elements, fabrication tolerances are usually around $1 \mathrm{~mm}$. Besides, wood-wood connections are not ideally rigid and gaps in the joints can add up through the structure causing large deviations and preventing the robot from assembling the pieces. Three strategies, which can be combined to ensure a precise insertion, have been identified in the literature and presented here.

\subsection{Self-centering connections}

A first method consists in adapting the design of the connections to enhance the tolerance and progressively guide the pieces to the final position. Usually the modification consists in chamfering one or both pieces or adding a separated guide.

Conic joints with a tolerance about $4 \mathrm{~cm}$ have for example been used in the FutureHome Project [8] in order to compensate for the swing of the automated crane, which was used to assemble the large modules. The slope of the cone was related to the friction forces between the different parts. In the extreme case of structures assembled by drones were precision is an even bigger challenge, specific joints have also been developed for masonry and timber elements [9]. Chamfering throughtenon joints is a commonly used technique in traditional woodworking. Such joints have notably been optimized for the insertion of the panels of the double-layered timber plate structure of the Vidy Theater [4].

A potential downside effect of self-centering connections is the diminution of the rigidity of the joint as the induced slopes are leading to smaller bending resistance.
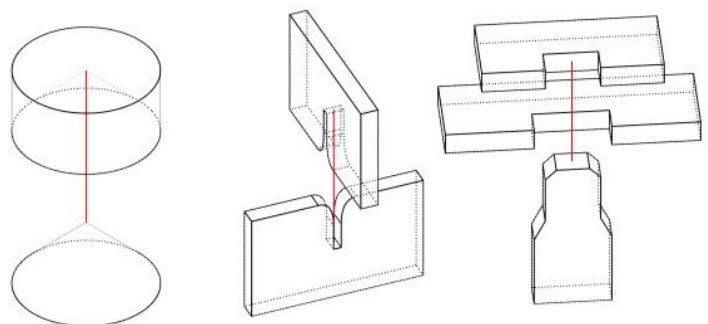

Figure 1. Three types of self-centering connections: conic joint (left), drone-compatible joint for interlocked timber beams (middle), and chamfered through-tenon joint (right).

\subsection{Force sensitive end effectors}

As manual insertion relies mostly on haptic feedback, another strategy is to use torque sensors to adapt the robot position according to the measured forces. A classic example consists in trying to insert a peg in a hole using integrated force sensors to align the robot position [10]. The need to manage material tolerances by developing robot sensitivity has also been illustrated in the DIANA project [11] where a robotic arm was interactively taught how to insert wooden rods to shape a ruled surface.

Impedance control has also been used for the insertion of gear-shaft mechanisms with a precision around $5 \mu \mathrm{m}$ for applications in medical fields or aeronautics [12]. More complex feedback loops using behavior-based or machine learning approaches have also been used to develop optimal strategies for inserting pieces into one another [13].

However, the interpretation of the measured force is always dependent of the shape and weight of the piece to insert and the above-mentioned techniques are established for standards symmetrical elements. Developing an adaptive strategy for the case of timber joinery is challenging as elements come in different sizes and the number and type of connections can also vary.

\subsection{Visual feedback}

Different position tracking systems based on visual feedback loops have been already developed for on-site applications and could also be applied off-site.

A total station can be used to track selected points and deduce the robot position by triangulation while another possibility is to rely on cameras and image recognition. A comparative study has highlighted the performance of fiducial markers for reducing deviation with a clear advantage regarding the execution speed [14]. Fiducial markers have also been used to guide the In situ Fabricator developed at ETH Zurich [15].

Other applications of visual detection includes precisely laying mortar on a brick wall [13] and assembling large modular frames [16] using a combination of cameras and lasers to guide the insertion. 
Photogrammetry and laser scanning technologies have also been combined with robotic arms to gather data on-site for indoor and outdoor localization [17][18][19]. However, image reconstruction, point cloud acquisition, and mesh post processing are all computationally intensive and data interpretation requires complex machine-learning algorithms in order to work with different geometries.

\section{Methodology}

\subsection{Integrated design framework}

Our approach consists in linking project design and technical constraints by means of computational geometry. Instead of locally solving the insertion problem, the goal is to inform the designer with fabrication and assembly constraints by developing a cross-platform workflow. Custom scripts are used to convey the geometric information between software.

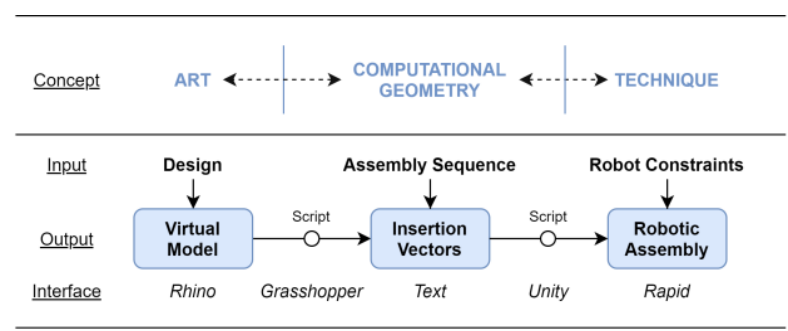

Figure 2. Integrated design framework linking design to robotics constraints.

The assembly is driven by three major inputs: design, assembly sequence, and robot constraints. In a traditional workflow each input is integrated one at a time in the construction process. Digitalization allows for more agility since parameters can be modified and information can flow back and forth.

It was decided to rely on existing specialized software for each part of the workflow instead of building a single custom program from scratch. Rhinoceros 6 (Robert McNeel \& Associates) was chosen as the design interface and the plugin Grasshopper as a tool to extract and manipulate data from the model. To simulate and execute the robot trajectory, the research benefitted from a collaboration with a specialized industrial partner, Imax Pro S.A. A custom application was developed, for the purpose of the research project, on the game engine Unity (Unity Technologies) to convert geometric data from text file to robot instructions.

Splitting the workflow between different software avoided making compromises between design possibilities and robotic performance. Meanwhile custom scripts ensured a smooth transition between the different interfaces allowing almost instantaneous feedback and testing of multiple design iterations.

\subsection{Insertion vectors}

Insertion vectors are an essential parameter for IATPS as the geometry of the joints is tightly connected to the assembly sequence. In fact, it is not possible to design the shape of the connectors without knowing the trajectory of the insertion beforehand. The integration of fabrication and assembly constraints follows an iterative process and is inherent to the design of IATPS.

A parametric script was thus coded inside the Grasshopper interface to deduce insertion vectors from a geometric input and a specific assembly sequence. Contact zones are identified by computing intersections between the panels while the type of joints that is generated depends on which faces are connected (e.g. through-tenon joints are created for a connection between the side of a panel and the face of its neighbor).

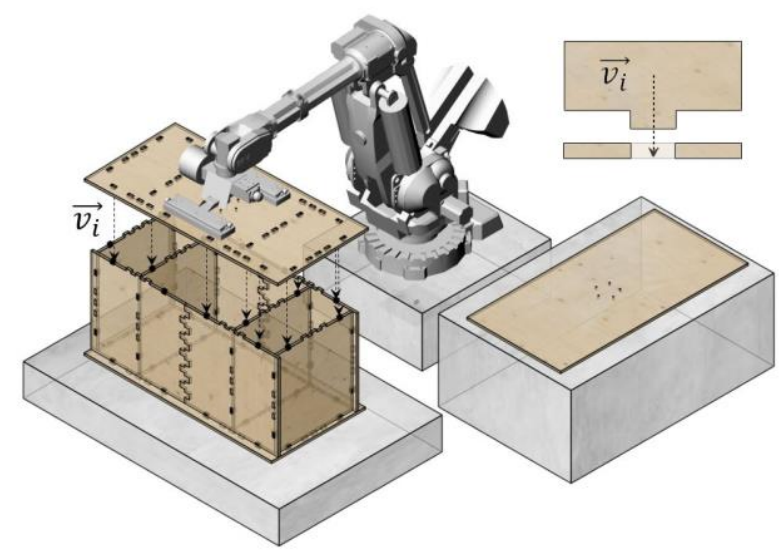

Figure 3. Insertion vectors are inducing the shape of the connections as well as the robot trajectory.

Once the 3D model is created, the contour of the panel can be extracted and the panels can be cut using a CNC. Then insertion vectors are used a second time to generate the robot trajectory, as further described in section 3.5.

\subsection{Position detection using fiducial markers}

Given the variety of possible configurations for IATPS, the use of fiducial markers was found to be the most efficient method to keep track of the position of the different timber panels. The open-source library OpenCV [20] provides a robust solution for estimating the position of fiducial markers (ArUco) from different sizes. A custom python executable was, therefore, developed to assess the performance of the detection before being integrated to the global workflow.

The algorithm consists in taking one picture from a targeted marker on a panel, computing its orientation and position coordinates and saving those results in a text file. This information is later accessed by the robot controller to update the trajectory. 
The image is processed by applying perspective transformation and thresholding to get potential markers from the pixels. Analyzing the color of the 36 cells composing the ArUco provides the unique identification number of the marker. The position and the orientation are obtained by finding the tridimensional transformation from the coordinate system of the camera to the coordinate system of the marker. Rotations are obtained in Euler angles but converted to quaternions to ease the conversion to Unity software, which is using another axes convention.

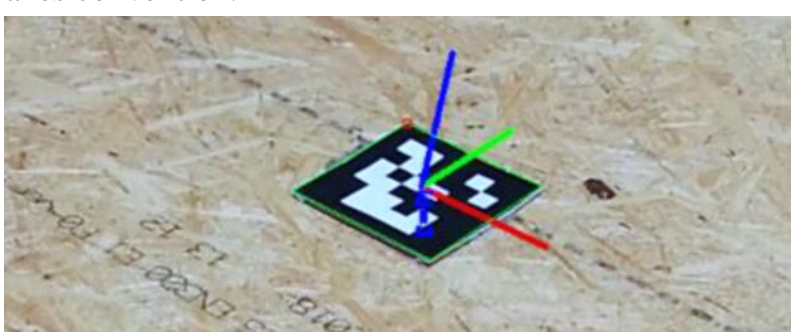

Figure 4. Image processing of an ArUco Marker using OpenCV library to get the position and orientation of a timber panel.

A key feature of the developed application is that the position of the different targets constituting the robot trajectory are expressed relatively to different coordinate systems. The position of some targets is associated to the coordinate system of the starting location while the position of some other targets is expressed relatively to the position and orientation of an ArUco marker. Hence, updating the position of this marker will automatically update the absolute coordinates of all associated targets while others will remain unchanged.
A unique marker has to be assigned to each panel and positioned at the center of gravity of the panel. Although the point of reference can be set arbitrarily, making it coincide with the point from which the tool would lift the panel, seems the most logical. Potential bending of the panel is minimized by picking it from its center of gravity.

Before proceeding to the robotic assembly, the position of the stack of panels is precisely referenced. Hence applying the visual feedback loop to adjust the starting location of the panels was not considered as necessary and only the end point of each trajectory is updated using fiducial markers. Prior to each pick and place operation, pictures of a marker, placed on the panels on which the insertion will occur, are taken with a camera mounted on the robotic arm (technical specifications are given in 4.1).

\subsection{Fail-safe process}

One drawback of working in relative with a feedback loop is that detection errors can potentially lead to unexpected trajectories, different from the simulation. Indeed, under certain circumstances, such as in case of insufficient luminosity or when the marker is too far or in the periphery of the angle of view of the camera, the precision of the detection significantly decreases.

In order to prevent updating the position of the marker with inaccurate coordinates, it was decided to take three pictures of each marker at different angles and set two tolerance parameters. If the dispersion of the results or the deviation from the model is too high, new pictures are taken and the updated values are again compared.

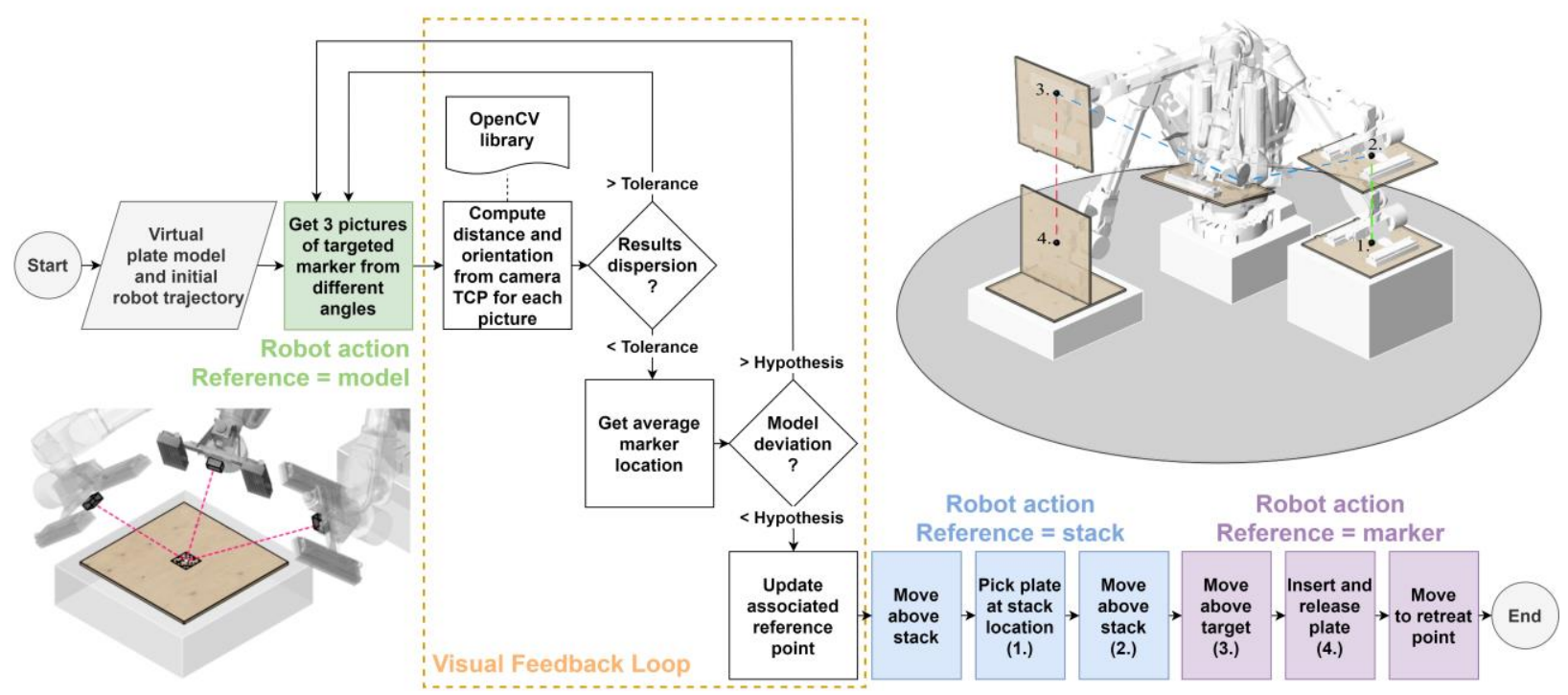

Figure 5. Robotic workflow: camera shooting with robot moving according to model (green), image processing (orange), picking phase with robot moving according to the stack of panels (blue), insertion phase with robot moving according to marker (purple). 


\subsection{Robotic Workflow}

Putting together the initial information given by the insertion vectors with the visual feedback loop, a complete workflow was established to insert the timber panels with a robotic arm (Figure 5). No path planning algorithm was used for the robot trajectory. Instead a strategic approach was preferred.

After the first panel of the sequence is placed, the robot positions itself above the marker using the information of the model as reference. The visual feedback loop is executed and pictures are taken from different angles at a distance of about $50 \mathrm{~cm}$. Then the position of the marker in the virtual model is updated through image processing.

The second step consists in picking the next panel at the stack location. As the position of the stack as well as the dimensions of the panels are known, the robot uses that information to move right above the stack and lift the panel from its center of gravity.

For the third step, the updated position of the fiducial marker is used as the new system of reference and a target is generated above the place of assembly. As a linear move is not always possible between those two position, a joint move is then preferred for this part of the trajectory. In order to constrain the interpolation additional targets can eventually be created in between.

For the last step, the insertion of the panel is finally performed (Figure 7). The robot reaches a target located a few centimeters away from the final position, in the opposite direction of the vector of insertion associated to the panel. Then it follows that vector until the panel is inserted. The panel is released by the vacuum gripper and the robot moves away along the normal to the panel surface before going back above the assembly. A structure is gradually assembled by repeating the process with the next panels.

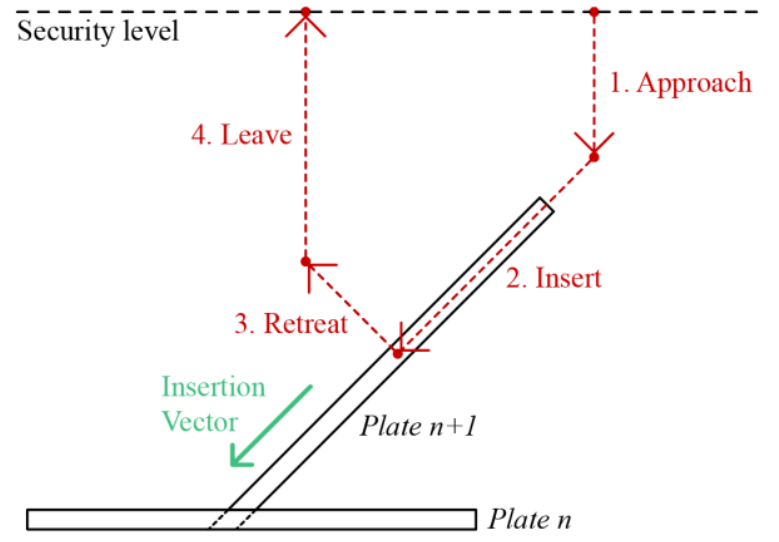

Figure 6. 2D representation of the assembly strategy based on the vector of insertion associated to the panel.

\subsection{Intermediate robot language}

Once established, the workflow was converted to robot instructions. Target positions and special actions such as taking a picture or activating the vacuum gripper were interpreted from a text file, which was manually typed or automatically generated by script (e.g. in the case of complex assemblies or when a high number of elements needs to be inserted). An intermediate programming language with a high level of abstraction was therefore developed in order to integrate custom commands.

Two examples of instructions are given in Figure 7. The number of parameters on each line depends on the first keyword: Camera will start the visual feedback loop and requires 4 additional parameters while JointMove requires up to 12 parameters including the coordinates of the targeted position to execute an unconstrained motion between the actual position of the robot and the specified point.

Other typical commands include LinearMove, which takes the same parameters as JointMove, Vacuum followed by $O n / O f f$, which is used to activate the suction of the gripper, and Wait followed by a number to pause the execution of the code during a certain amount of time.

Finally, each line of the code is parsed by our application in Unity for simulation, and converted to the specific programming language of the robot for execution. On a side note, the additional layer of abstraction added by this intermediate language proved to significantly enrich the user-experience by providing an explicit workflow.

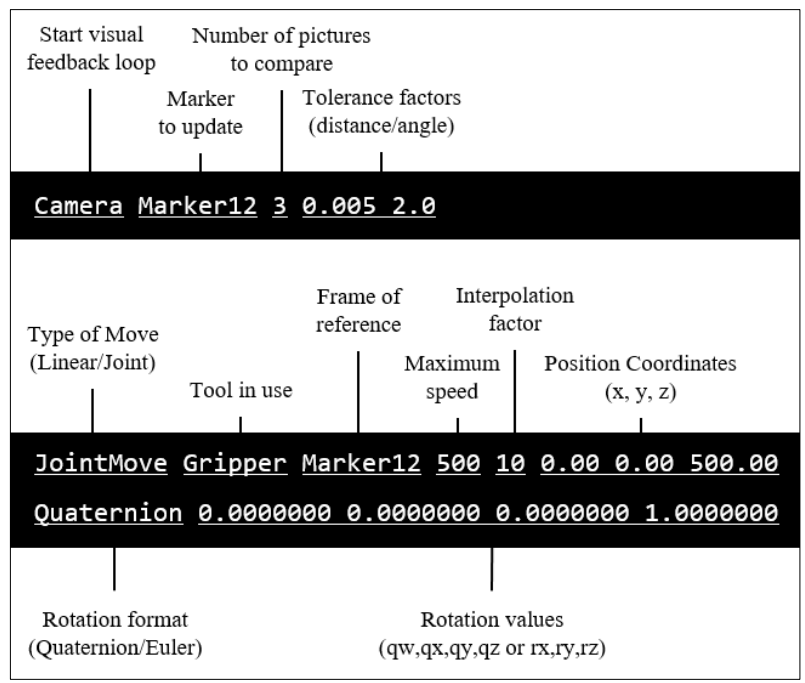

Figure 7. Samples of a text file interpreted by our custom application in Unity to send instructions to the robot controller: Activation of the visual feedback loop (top) and joint move above a marker (bottom). 


\section{Tests and results}

\subsection{Experimental set up}

Experiments were led with a 6-axis robotic arm (ABB IRB 6400R) with a reach of $2.5 \mathrm{~m}$. The end effector was equipped with two vacuum grippers, which can lift panels up to $80 \mathrm{~kg}$. In addition, a standard webcam (Logitech C270) with a resolution of 1 megapixel was mounted on top of the robot effector and connected by USB cable in order to take pictures of the ArUco markers. Calibration was carried out by taking a series of 20 pictures of an ArUco board from different angles to get the intrinsic parameters of the camera such as the focal distances and the center of the camera. Fiducial markers were then printed as $10 \mathrm{~cm}$ square and precisely fixed to the center of the panels following guiding lines previously engraved with a CNC.

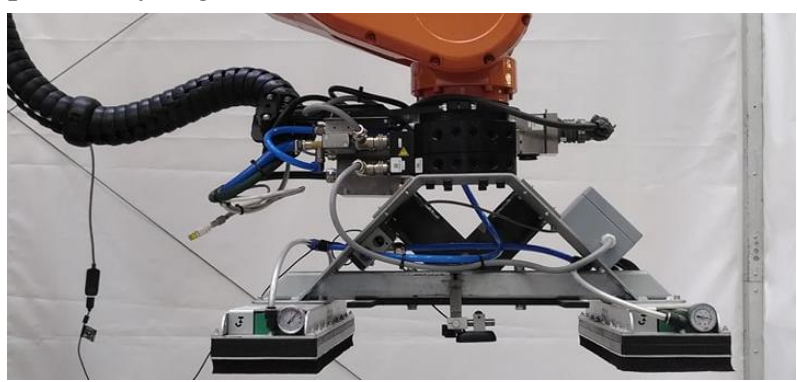

Figure 8. End-effector of the robot equipped with a vacuum gripper and camera for visual feedback.

\subsection{Insertion without visual feedback loop}

A first test was led without the visual feedback loop to evaluate the difficulty of inserting panels with a robotic arm. The objective was to automate the assembly of 3 non-orthogonal timber boxes composed of 13 panels of $45 \mathrm{~mm}$ thick cross laminated timber (CLT). The gap between the tenon and the mortise was set to $0.5 \mathrm{~mm}$ to reduce friction forces and reached up to $0.7 \mathrm{~mm}$ in some cases due to slight material deformations in addition to the tolerance of fabrication.

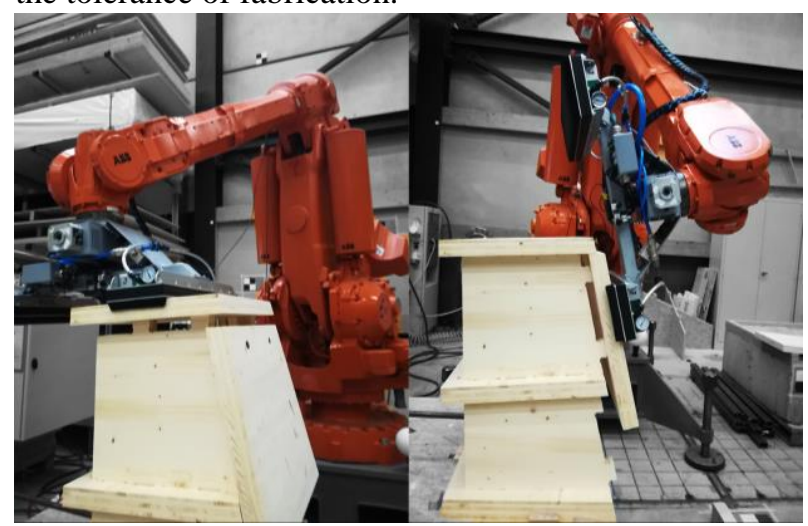

Figure 9. Assembly of 13 non-orthogonal timber panels without using the visual feedback loop.
The accumulation of small discrepancies due to the tolerance in the joints and the dimensional variation of the panels caused large deviations between the virtual model and the physical prototype. When inserting the last panel, a difference of about $1 \mathrm{~cm}$ was measured at the top of structure. This could be explained by the fact that only the first panel was anchored while the rigidity of the connections for the other panels was not enough to prevent them from slightly rotating. This led to the impossibility of assembling the pieces without a manual intervention. In conclusion, as inaccuracy increases with the number and the size of the elements in the structure, a visual feedback loop was found to be necessary.

\subsection{Precision of the visual feedback loop}

Prior to testing the insertion of panels, the accuracy of the visual feedback was evaluated. The position of the camera in relation to the end effector of the robotic arm was found by manually referencing the position of 4 markers (Figure 10) and matching the values obtained by taking a picture. It is assumed that both the calibration of the sharp tool and the manual referencing of the markers was achieved with a precision of about $0.5 \mathrm{~mm}$.

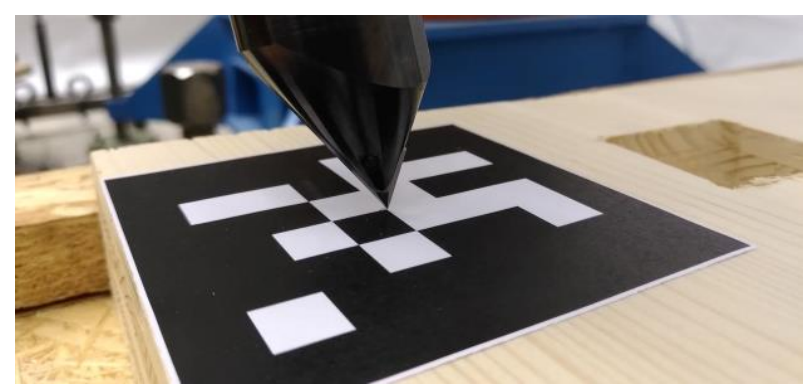

Figure 10. Finding relative camera position by comparing manually referenced coordinates with computed values from the visual feedback.

Once the position of the camera was properly set up, new pictures were taken with multiple camera orientations and distances and the acquired data was compared with the coordinates of the manually referenced markers. Three pictures were taken at each location to assess the consistency of the results.

Extreme values for distances of 30,50 and $80 \mathrm{~cm}$ between the camera and the fiducial marker are reported in Figure 11. Both precision and accuracy were affected by the distance from which the picture was taken. However, below $30 \mathrm{~cm}$, the dispersion of the results stayed below $1 \mathrm{~mm}$, which stays in the range of precision of the manual measurements. Further than $50 \mathrm{~cm}$, the accuracy of the visual detection was decreasing considerably reaching several millimeters at $80 \mathrm{~cm}$. In addition, a loss of precision was also observed as the dispersion of the results which were obtained from the same camera position raised to $2 \mathrm{~mm}$ at $80 \mathrm{~cm}$. 


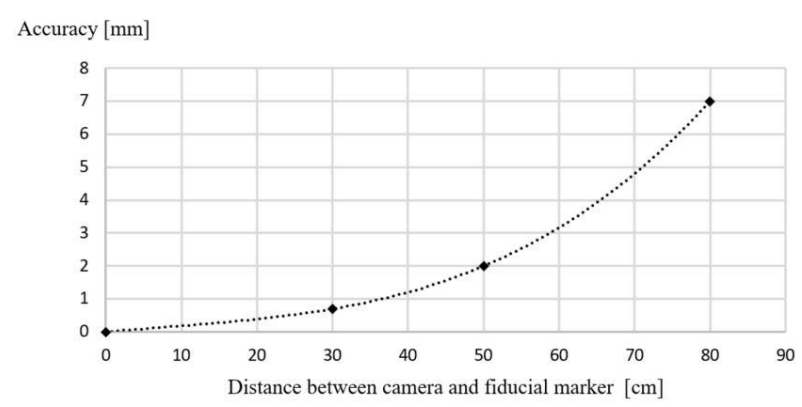

Figure 11. Maximum distance between target position and the results obtained with the visual feedback loop.

Additional tests were performed with different orientations of the robot end-effector and also produced slight deviations from the referenced point. Targets on the edge of the field of view of the camera were particularly misinterpreted by the algorithm and reported position errors up to $2 \mathrm{~cm}$. On one hand, the calibration of the camera was found to be a key parameter to reach more accurate results but was, at the same time, limited by the precision of the manual measurements. On the other hand, the precision of the detection was also limited by the algorithm itself and the exactitude of the spatial transformation.

\subsection{Insertion with visual feedback loop}

Based on the previous results, a tolerance threshold was established. It was shown that all pictures taken at a distance below $50 \mathrm{~cm}$, with the camera parallel to the marker, were interpreted with a maximum error of $3 \mathrm{~mm}$. The design of the timber joints was therefore adapted in consequence to match that tolerance threshold as shown in Figure 12.

A test of insertion was conducted using two panels with 1 square meter each connected by two trough-tenon joints. Following the principles of auto-centering connections, a chamfer of $4 \mathrm{~mm}$ was applied to tenons and mortises. As the fabrication process excluded cutting the panel from below, the chamfer was doubled on top of the tenon instead of being equally distributed. Using the visual feedback loop, the two panels could finally be inserted into one another (Figure 13).

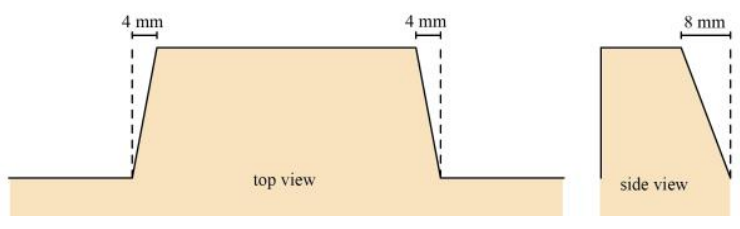

Figure 12. Chamfered tenon based on the measured precision of the visual feedback loop.

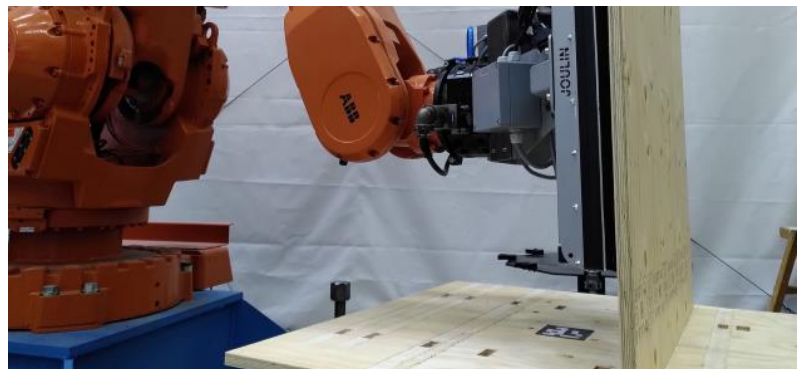

Figure 13. Insertion of a laminated veneer lumber (LVL) panel with the visual feedback loop.

\section{Conclusion and outlook}

A complete workflow was established to assemble prefabricated timber panels connected by wood-wood connections with a robotic arm. The study included the development of a strategy of insertion using different software and linking design and assembly constraints by the means of computational geometry. In addition, an explicit programming language was introduced to ease the interactions between designer and machine.

A particular focus was set on performing a precise insertion despite the large deviations that might occur between physical prototypes and virtual models. The visual detection of fiducial markers, which can easily be placed on top of timber plates, was found to be a cheap and effective solution for updating the position of the panels through a feedback loop.

The evaluation of the method performance showed satisfying results at distances up to $50 \mathrm{~cm}$. For larger distances, the precision of the detection of the markers showed some limitations but could probably be enhanced by refining camera calibration. Eventually, increasing the camera resolution or the size of the ArUco markers could improve the quality of image processing and lead to better outcomes.

Finally, applying the visual feedback loop to the assembly of two timber plates demonstrated the potential of the concept and the feasibility of the proposed workflow in order to automate the assembly of Integrally Attached Timber Plate Structures. The method presented in this paper could have applications on-site. However, further research is still required to extend the workflow to the architectural scale such as solving issues related to friction when inserting multiple joints at the same time.

\section{Acknowledgement}

This research was supported by the NCCR Digital Fabrication, funded by the Swiss National Science Foundation (NCCR Digital Fabrication Agreement \#51NF40-141853). In addition, the authors would also like to acknowledge Imax Pro S.A. for the technical support regarding the integration of the robotic system. 


\section{References}

[1] United Nations. Sustainable Development Goals. Online: https://sustainabledevelopment.un.org/, Accessed: 18/05/2020.

[2] Churkina G. Organschi A. Reyer C. Ruff A. Vinke K. Liu Z. Reck B. Graedel T.E. Schellnhuber H.J. Buildings as a global carbon sink. Nature Sustainability, 3, 269-276, 2020.

[3] Robeller C. Integral Mechanical Attachment for Timber Folded Plate Structures. EPFL, 2015.

[4] Robeller C. Gamerro J. Weinand Y. Théâtre Vidy Lausanne - A double-layered timber folded plate structure. Journal of the International Association for Shell and Spatial Structures, 58(4):295-314, 2017.

[5] Alvarez M. Wagner H.J. Groenewolt A. Krieg O.D. Kyjanek O. Sonntag D. Bechert S. Aldinger L. Menges A. and Knippers J. Integrative interdisciplinary advancements of digital timber architecture - The BUGA Wood Pavilion. In Proceedings of the Association for Computer Aided Design in Architecture, Austin, United States, 2019.

[6] Nguyen A.C. Vestartas P. Weinand Y. Design framework for the structural analysis of freeform timber plate structures using wood-wood connections. Automation in Construction, 107, 2019.

[7] Robeller C. Weinand Y. Helm V. Thoma A. Gramazio F. Kolher H. Robotic Integral Attachment. Fabricate, 2017.

[8] Balaguer C. Abderrahim M. Navarro J.M. Boudjabeur S. Aromaa P. Kahkonen K. Slavenburg S. Seward D. Bock T. Wing R. and Atkin B. FutureHome: An integrated construction automation approach. Robotics and Automation Magazine, 9(1):55-66, 2002.

[9] Goessens S. Rogeau N. De Beusscher G. Mueller C. Latteur P. Parametric Design of DroneCompatible Architectural Timber Structures. In Proceedings of the International Association for Shell and Spatial Structures (IASS), Boston, United States, 2018.

[10] Strip D.R. Technology for Robotic Mechanical Assembly: Force - Directed Insertions. AT\&T Technical Journal, 67(2):23-34, 1988.

[11] Odehnal B. Hornung P. Santorso K. Ambrosz B. Sampl G. Golob E. Brell-Cokçan S. Braumann J. Cokçan B. Robotic Woodcraft - Towards The
Craftmanship Of The Future. University of Applied Arts Vienna, 2019.

[12] Guo P. Zhang Z. Liu Y. Sun W. Li Z. Precision assembly method based on coaxial alignment and force control. Journal of Physics: Conference Series, 1303, 2019.

[13] Elashry K. Glynn R. An Approach to Automated Construction Using Adaptive Programing. In Proceedings of Robotic Fabrication in Architecture, Art and Design, pages: 51-66, 2014.

[14] Iturralde K. Kinoshita T. Bock T. Grasped Element Position Recognition and Robot Pose Adjustment during Assembly. In Proceedings of 36th International Symposium on Automation and Robotics in Construction, pages: 461-468, Banff, Canada, 2019.

[15] Giftthaler M. Sandy T. Dörfler K. Brooks I. Buckingham M. Rey G. Kohler M. Gramazio F. Buchli J. Mobile robotic fabrication at 1:1 scale: the In situ Fabricator System, experiences and current developments. Construction Robotics, 1:3-14, 2017.

[16] Qin Z. Wang P. Sun J. Lu J. Qiao H. Precise Robotic Assembly for Large-Scale Objects Based on Automatic Guidance and Alignment. IEEE Transactions on Instrumentation and Measurement, 65:1398-1411, 2016.

[17] Vincke S. Bassier M. Vergauwen M. Image Recording Challenges for Photogrammetric Construction Site Monitoring. ISPRS Annals of the Photogrammetry, Remote Sensing and Spatial Information Sciences, pages: 747-753, 2019.

[18] Kim P. Chen J. Cho Y.K. Autonomous mobile robot localization and mapping for unknown construction environments. Construction Research Congress, pages: 147-156, 2018

[19] Gawel A. Blum H. Pankert J. Krämer K. Bartolomei L. Ercan S. Farshidian F. Chli M. Gramazio F. Siegwart R. Hutter M. Sandy T. A Fully-Integrated Sensing and Control System for High-Accuracy Mobile Robotic Building Construction. International Conference on Intelligent Robots and Systems (IROS), 2019.

[20] Garrido-Jurado S. Muñoz-Salinas R. MadridCuevas F.J. Marín-Jiménez M.J. Automatic generation and detection of highly reliable fiducial markers under occlusion, Pattern Recognition, 47:2280-2292, 2014. 